\title{
A NOTE ON ENTROPY INEQUALITIES AND ERROR ESTIMATES FOR HIGHER-ORDER ACCURATE FINITE VOLUME SCHEMES ON IRREGULAR FAMILIES OF GRIDS
}

\author{
SEBASTIAN NOELLE
}

\begin{abstract}
Recently, Cockburn, Coquel and LeFloch proved convergence and error estimates for higher-order finite volume schemes. Their result is based on entropy inequalities which are derived under restrictive assumptions on either the flux function or the numerical fluxes. Moreover, they assume that the spatial grid satisfies a standard regularity assumption. Using instead entropy inequalities derived in previous work by Kröner, Noelle and Rokyta and a weaker condition on the grid, we can generalize and simplify the error estimates.
\end{abstract}

\section{INTRODUCTION}

In a recent paper, Cockburn, Coquel and LeFloch [2] derived error estimates for higher-order finite volume schemes for scalar conservation laws

$$
\begin{aligned}
\partial_{t} u+\operatorname{div} f(u)=0 & \text { in } \mathbf{R}_{+} \times \mathbf{R}^{d}, \\
u(0)=u_{0} & \text { on } \mathbf{R}^{d} .
\end{aligned}
$$

They modified the Kuznetsov [7] approximation theory to obtain an $h^{1 / 4}$ convergence rate. Analogous results were derived by Vila [10] for first-order finite volume schemes and by Cockburn and Gremaud [4] for the streamline diffusion shockcapturing and the discontinuous Galerkin finite element methods.

In order to derive the error estimate, Cockburn, Coquel and LeFloch assume that the entropy inequality

$$
\begin{gathered}
U_{M}\left(u_{K, e}^{n+1}, c\right)-U_{M}\left(u_{K}^{n}, c\right)+\frac{\tau p_{K}}{|K|}\left\{G_{e, K}^{n}-F_{M}\left(u_{K}^{n}, c\right) \cdot N_{e, K}\right\} \\
\leq \frac{\tau p_{K}}{|K|} a_{K, e}^{n} \frac{\partial U_{M}}{\partial v}\left(u_{K, e}^{n}, c\right)
\end{gathered}
$$

holds together with the bound

$$
\sum_{0 \leq n \tau \leq T} \sum_{K \in \mathcal{T}_{h}} \sum_{e \subset \partial K}\left|a_{K, e}^{n}\right|\left|u_{K, e}^{n}-u_{K_{e}, e}^{n}\right||e| \tau \leq C_{1} h^{\alpha},
$$

where $\alpha>0$ is a fixed constant (for the notation compare [2]). For finite volume schemes built on a piecewise constant approximation and E-fluxes, the entropy

Received by the editor March 21, 1995.

1991 Mathematics Subject Classification. Primary 35L65, 65M12, 65M15, 65M50.

Key words and phrases. Multidimensional conservation law, finite volume method, discrete entropy inequality, error estimate, irregular grids.

Partially supported by Deutsche Forschungsgemeinschaft, SFB 256. 
inequality (3) holds naturally with right-hand side equal to zero. For schemes which use piecewise polynomial approximations, additional assumptions are needed in order to guarantee (3) and (4). Cockburn, Coquel and LeFloch derive such entropy inequalities for antidiffusive schemes, which are assumed to satisfy so-called "sharp entropy inequalities". For the higher-order schemes, these "sharp entropy inequalities" are derived in $[3,5]$ under the assumption that either

(i) there is a $\delta>0$ such that for all $u$ and all $N_{e, K}$

$$
\left|\frac{\partial^{2} f(u)}{\partial u^{2}} \cdot N_{e, K}\right| \geq \delta
$$

or

(ii) the numerical viscosity coefficient of the scheme is bounded below by a positive constant.

Condition (i) restricts both the grids and the flux functions: given any vector $\partial^{2} f(u) / \partial u^{2}$, there are always directions $N$ such that (5) is violated, so polygons with such normals have to be excluded from the grid. This may lead to complications, especially when the grid is refined adaptively. Moreover, certain flux functions cannot be treated by this approach, for example $f(u)=(\cos u, \sin u)$.

Condition (ii) excludes some important numerical flux functions, such as Godunov's flux and the Engquist-Osher flux.

We remark in this note that the main result of [2] holds if we replace (3), (4) by the assumption that

$$
U_{M}\left(u_{K, e}^{n+1}, c\right)-U_{M}\left(u_{K}^{n}, c\right)+\frac{\tau p_{K}}{|K|}\left\{G_{e, K}^{n}-F_{M}\left(u_{K}^{n}, c\right) \cdot N_{e, K}\right\} \leq C M h^{2 \alpha}
$$

where $\alpha \in\left(\frac{1}{2}, 1\right]$ is a given constant.

The point of this note is that the entropy inequality (6) has been obtained by the author [8] (see also [6]) for higher-order finite volume schemes without making any assumption of genuine nonlinearity on the flux function $f$ as in condition (i) above and for MUSCL-type finite volume schemes based on general E-fluxes, where condition (ii) is not required. Moreover, (6) may be obtained by one direct calculation (see [8, Lemma 4.3] or [6, Theorem 5.1]), while in order to derive (3), (4), one needs to combine the results of several sections of the papers $[5,3,2]$.

In [8], the author generalized existing convergence results for finite volume schemes to schemes built upon irregular families of grids, where cells may become flat at a certain rate as the grid parameter $h$ tends to zero. Such grids may be important in applications, especially when refining the grid along an essentially one-dimensional shock front or in a boundary layer. Here we refine the analysis of [2], made under a regularity assumption on the grid, to include irregular families of grids, and give precise estimates for the convergence rate.

In $\S 2$, we define the class of finite volume schemes considered in this paper and comment on our entropy inequality (6), and in $\S 3$, we sketch how to combine (6) with the analysis in [2] in order to obtain the error estimates. Since our paper is closely related to [2], we follow the notation of that paper, with the following exceptions: the constants $\alpha, \beta, \gamma, \delta, C_{1}$ and $C_{2}$ introduced here are not identical with those used in [2]. 


\section{Entropy inequalities For MUSCL-type FInite VOlume SChEMES}

Let $\mathcal{T}_{h}$ be a polygonal grid of $\mathbf{R}^{d}$. Given a polygon $K \in \mathcal{T}_{h}$, let $e \subset \partial K$ denote a side of $K$ and let $N_{e, K}$ denote the outward unit normal to $K$ at side $e$. We assume that the intersection of two polygons is a union of common sides and vertices. Let

$$
p_{K}:=\sum_{e \subset \partial K}|e|
$$

be the perimeter of $K$, let $h_{K}$ be the outer and $\rho_{K}$ the inner diameter of $K$. Let

$$
\begin{aligned}
h & :=\sup _{K \in \mathcal{T}_{h}} h_{K}, \\
\rho & :=\inf _{K \in \mathcal{T}_{h}} \rho_{K}
\end{aligned}
$$

and let $\tau>0$ be the timestep. Let the time $T>0$ be arbitrarily large and fixed, and let $t_{n}:=\tau n, n=0, \ldots, n_{T}$, such that $T=\tau n_{T}$. We consider schemes of the form

$$
u_{K}^{n+1}:=u_{K}^{n}-\frac{\tau}{|K|} \sum_{e \subset \partial K}|e| g_{e, K}^{n}=\sum_{e \subset \partial K} \frac{|e|}{p_{K}} u_{K, e}^{n+1}
$$

where

$$
u_{K, e}^{n+1}:=u_{K}^{n}-\frac{\tau p_{K}}{|K|}\left(g_{e, K}^{n}-f\left(u_{K}^{n}\right) \cdot N_{e, K}\right)
$$

and

$$
u_{K}^{0}:=\frac{1}{|K|} \int_{K} u_{0}(x) d x .
$$

The initial data $u_{0}$ are bounded and measurable, and for simplicity of exposition, we assume that $u_{0}$ has compact support.

The numerical flux in (7), (8) is defined by

$$
g_{e, K}^{n}:=g_{e, K}\left(u_{K}^{n}+\tilde{u}_{K, e}^{n}, u_{K_{e}}^{n}+\tilde{u}_{K_{e}, e}^{n}\right) .
$$

Here, $K_{e}$ is the neighbor of $K$ with $e \subset \partial K \cup \partial K_{e}$, and $g_{e, K}(\cdot, \cdot)$ is a two-point conservative $E$-flux consistent with $f \cdot N_{e, K}$, i.e., it satisfies

$$
\frac{g_{e, K}(v, w)-f(s) \cdot N_{e, K}}{w-v} \leq 0
$$

for all $s$ between $v$ and $w$ (compare Osher [9]). We assume that $f$ and $g$ possess global Lipschitz constants $L_{f}$ and $L_{g}$. Since we will only consider bounded initial data, this can always be achieved by modifying $f$ outside of the range of $u_{0}$. In general, $L_{g} \geq L_{f}$, but in practice, we may restrict our attention to schemes satisfying $L_{g}=L_{f}$, which is, for example, true for the Godunov flux and the modified Lax-Friedrichs flux (see $[5,8]$ ).

The values $\tilde{u}_{K, e}^{n}$ allow for a piecewise higher-order polynomial interpolation of the cell averages $u_{K}^{n}$ (compare the discussion in $[2,5,6,8]$ ). In the following we assume that there are constants $\alpha>0$ and $C_{1}, C_{2}$ independent of $h, T$ and $u_{0}$ such that

$$
\left|\tilde{u}_{K, e}^{n}\right| \leq C_{1} h^{\alpha}
$$

and

$$
\tilde{u}_{K, e}^{n}\left(u_{K}^{n}-u_{K_{e}}^{n}\right) \leq C_{2} h^{2 \alpha} .
$$


If $\alpha \leq 1$, then (11) and (12) do not reduce the formal order of accuracy of the scheme. Assumption (12) is a natural one: in the presence of large jumps, where $\left|u_{K}^{n}-u_{K_{e}}^{n}\right|>C_{1} h^{\alpha}$, it states that up to terms of order $h^{2 \alpha}$, the interpolated value $u_{K}^{n}+\tilde{u}_{K, e}^{n}$ at an edge $e$ between the cells $K$ and $K_{e}$ lies between the mean values $u_{K}^{n}$ and $u_{K_{e}}^{n}$ over these cells. Such an assumption is usually enforced by a flux-limiter.

On the space-time grid we make the assumptions that the standard CFL-condition

$$
\frac{\tau p_{K}}{|K|} L_{g} \leq 1
$$

holds for all $K \in T_{h}$ and that there are constants $\gamma>0$ and $C_{3}$ independent of $h$, $T$ and $u_{0}$ such that

$$
\frac{h^{2 \alpha}}{\tau} \leq C_{3} h^{\gamma}
$$

In fact, the timestep restriction (13) may be slightly relaxed by refining the convex decomposition $(7)$ - (8) of the numerical scheme and taking the local speed of wave propagation into account (see Appendix B of [8]).

Note that from (13), $\tau \leq C h$, so (14) is compatible with (13) only if $\gamma \leq 2 \alpha-1$. It is argued below that the timestep $\tau$ may be chosen to be of the order of $\rho$. Therefore, condition (14) weakens the standard regularity condition

$$
\frac{h}{\rho} \leq C .
$$

This is discussed in detail after the proof of Theorem 3.1.

Finally, we denote by $u^{h}: \mathbf{R}^{d} \times[0, T] \rightarrow \mathbf{R}$ the approximate solution, given as

$$
u^{h}(t, x):=u_{K}^{n} \text { for } x \in K, t \in\left[t_{n}, t_{n+1}\right) .
$$

Under these assumptions, it can be verified immediately that $u^{h}$ satisfies the $L^{\infty}$ bound

$$
\left\|u^{h}\right\|_{L^{\infty}\left(\mathbf{R}^{d} \times[0, T]\right)} \leq\left\|u_{0}\right\|_{L^{\infty}\left(\mathbf{R}^{d}\right)}+C T h^{\gamma-\alpha} .
$$

Here and below we use $C$ for constants which do not depend on $h, T$ and $u_{0}$.

In $[6,8]$ Kröner, Rokyta and the author showed that the discrete entropy inequality (6) holds for the Godunov and the Lax-Friedrichs flux. Since all $E$-fluxes are convex combinations of these two fluxes, (6) holds for all MUSCL-type finite volume schemes built on $E$-fluxes (see [8]).

\section{ERror Estimates}

As in [2], we assume that the scheme has a finite speed of propagation. In particular, we assume that there are compact subsets $\Omega_{h}(t)$ of $\mathbf{R}^{d}, 0 \leq t \leq T$, such that

$$
\Omega_{h}\left(t_{1}\right) \subset \Omega_{h}\left(t_{2}\right) \subset \subset \mathbf{R}^{d} \text { for } 0 \leq t_{1} \leq t_{2} \leq T
$$

and

$$
\operatorname{supp} u(t, \cdot) \cup \operatorname{supp} u^{h}(t, \cdot) \subset \Omega_{h}(t) \quad \text { for } \quad 0 \leq t \leq T,
$$

where $u$ is the entropy weak solution of (1), (2). 
Following Kuznetsov [7], Cockburn, Coquel and Le Floch show that

$$
\begin{aligned}
& \left\|u^{h}(T, \cdot)-u(T, \cdot)\right\|_{L^{1}\left(\mathbf{R}^{d}\right)} \\
& \leq \leq \frac{\left|\Omega^{h}(T)\right|}{2 M}+3\left\|u^{h}(0, \cdot)-u_{0}\right\|_{L^{1}\left(\mathbf{R}^{d}\right)}+9\left(\varepsilon+\varepsilon_{0}\left\|\frac{\partial f}{\partial u}\left(u_{0}\right)\right\|_{L^{\infty}\left(\mathbf{R}^{d}\right)}\right) T V\left(u_{0}\right) \\
& \quad+6 \sup _{0 \leq t \leq T}\left(S_{\varepsilon_{0}, \varepsilon}^{h}\left(u, u^{h} ; t\right)+E_{\varepsilon_{0}, \varepsilon}^{h}\left(u, u^{h} ; t\right)+E_{\varepsilon_{0}, \varepsilon}^{h}\left(u^{h}, u ; t\right)\right) .
\end{aligned}
$$

Here, $T V\left(u_{0}\right)$ denotes the total variation of $u_{0}$. For the definition of $S_{\varepsilon_{0}, \varepsilon}^{h}$ and $E_{\varepsilon_{0}, \varepsilon}^{h}$, compare [2]. Briefly, $\varepsilon_{0}>0$ and $\varepsilon>0$ are small parameters defining a family of test functions $\omega_{\varepsilon_{0}}(t)$ and $\psi_{\varepsilon}(x)$, and the large parameter $M$ regularizes the Kružkov entropy $U(u, c):=|u-c|$ and the corresponding entropy flux $F(u, c)$. Moreover, $S_{\varepsilon_{0}, \varepsilon}^{h}\left(u, u^{h} ; t\right)$ is the lack of symmetry of the entropy pairs $\left(U_{M}, F_{M}\right), E_{\varepsilon_{0}, \varepsilon}^{h}\left(u, u^{h} ; t\right)$ is the entropy production of the entropy weak solution $u$ and $E_{\varepsilon_{0}, \varepsilon}^{h}\left(u^{h}, u ; t\right)$ is the entropy production of the approximate solution $u^{h}$.

Under the assumption that $\varepsilon \geq h$, and using the $L^{\infty}$-bound (17), one can show precisely as in [2] that there is a constant $C>0$ such that for $0 \leq t \leq T$

$$
\begin{aligned}
S_{\varepsilon_{0}, \varepsilon}^{h}\left(u, u^{h} ; t\right)+E_{\varepsilon_{0}, \varepsilon}^{h}\left(u, u^{h} ; t\right) \leq & C \frac{1}{M} T V\left(u_{0}\right) T+C \frac{h^{1+\gamma-\alpha}}{\varepsilon} T\left|\Omega_{h}(T)\right| \\
& +C \frac{h}{\varepsilon}\left(T V\left(u_{0}\right) T+\left\|u_{0}\right\|_{L^{\infty}\left(\mathbf{R}^{d}\right)}\left|\Omega_{h}(T)\right|\right) .
\end{aligned}
$$

The term $E_{\varepsilon_{0}, \varepsilon}^{h}\left(u^{h}, u ; t\right)$ is the one which is the most difficult to handle, and it is here where Cockburn, Coquel and LeFloch use their entropy inequalities (3), (4). In the following, we briefly sketch how to obtain an error estimate analogous to that in [2] when replacing (3), (4) and the regularity assumption (15) by our estimate (6) and the assumption (14). For details, we refer the reader to [2].

First, one can use (6) to derive the auxiliary estimate

$$
\sum_{n=0}^{n_{T}-1} \sum_{K \in \mathcal{T}_{h}} \sum_{e \subset \partial K} \frac{|e||K|}{p_{K}}\left|u_{K, e}^{n+1}-u_{K}^{n+1}\right|^{2} \leq\left\|u_{0}\right\|_{L^{2}}^{2}+C \frac{h^{2 \alpha}}{\tau} T\left|\Omega_{h}(T)\right| .
$$

Modifying the derivation in [2] slightly, we obtain

$$
E_{\varepsilon_{0}, \varepsilon}^{h}\left(u^{h}, u ; t\right) \leq \sum_{j=1}^{3} \int_{Q_{T}} \theta_{j}(t, x, u(t, x)) d t d x+\int_{Q_{T}} \theta_{4}(t, x) d t d x .
$$

Here, $\theta_{1}, \theta_{2}$ and $\theta_{3}$ are defined as in [2], and

$$
\theta_{4}(t, x):=C M h^{2 \alpha} \sum_{n=0}^{n_{T}-1} \sum_{K \in \mathcal{T}_{h}}|K| \tilde{\psi}_{\varepsilon}^{\prime}\left(x ; x^{\prime}\right) \omega_{\varepsilon_{0}}\left(t_{n+1}^{\prime}-t\right),
$$

where the test functions $\tilde{\psi}_{\varepsilon}^{\prime}$ and $\omega_{\varepsilon_{0}}$ are defined in [2]. One can now obtain the estimates

$$
\int_{Q_{T}} \theta_{1}(t, x, u(t, x)) d t d x \leq C \frac{h}{\varepsilon \tau^{1 / 2}}\left\|u_{0}\right\|_{L^{2}} T^{1 / 2}\left|\Omega_{h}(T)\right|^{1 / 2}+C \frac{h^{1+\alpha}}{\varepsilon \tau^{1 / 2}} T\left|\Omega_{h}(T)\right|,
$$




$$
\begin{aligned}
& \int_{Q_{T}}\left\{\theta_{2}(t, x, u(t, x))+\theta_{3}(t, x, u(t, x))\right\} d t d x \leq C \frac{h}{\varepsilon}\left\|u_{0}\right\|_{L^{\infty}\left(\mathbf{R}^{d}\right)}\left|\Omega_{h}(t)\right| \\
&+C \frac{h^{1+\gamma-\alpha}}{\varepsilon} T\left|\Omega_{h}(t)\right|, \\
& \int_{Q_{T}} \theta_{4}(t, x) d t d x \leq C M h^{2 \alpha-1} T\left|\Omega_{h}(T)\right| .
\end{aligned}
$$

Now the following convergence result is basically a corollary of the proof of Theorem 2.1 in $[2]$.

Theorem 3.1. Let $u_{0} \in L^{\infty}\left(\mathbf{R}^{d}\right) \cap B V\left(\mathbf{R}^{d}\right)$ be of compact support and let $T>0$ be given. Let $u$ be the entropy weak solution of (1), (2) and $u_{h}$ the approximate solution defined by the (possibly higher-order) finite volume scheme (7) - (10) and (16). Suppose that $1 / 2<\alpha \leq 1$ and $0<\gamma \leq 2 \alpha-1$ are given independently of $h$ and that conditions (11), (12), (13), (14), (18) and (19) hold. Then there are positive constants $\delta, h_{0}$ and $C_{0}$ such that for all $t \in[0, T]$ and all $h \in\left(0, h_{0}\right]$ the approximate solution $u^{h}$ satisfies the error estimate

$$
\left\|u^{h}(t, \cdot)-u(t, \cdot)\right\|_{L^{1}\left(\mathbf{R}^{d}\right)} \leq C_{0} h^{\delta} .
$$

Here, $\delta$ is independent of $h, T$ and $u_{0}$, while $h_{0}$ and $C_{0}$ may depend on $T,\left|\Omega_{h}(T)\right|$, $T V\left(u_{0}\right),\left\|u_{0}\right\|_{L^{\infty}\left(\mathbf{R}^{d}\right)}$ and $\left\|u_{0}\right\|_{L^{2}\left(\mathbf{R}^{d}\right)}$, but not on $h$. In particular,

(i) if $3 / 4 \leq \alpha \leq 1$ and $\gamma=2 \alpha-1$, then

$$
\delta=\frac{1}{4}
$$

(ii) if $3 / 4<\alpha \leq 1$ and $2(1-\alpha) \leq \gamma<2 \alpha-1$, then

$$
\delta=\frac{1+\gamma / 2-\alpha}{2}<\frac{1}{4}
$$

(iii) if $1 / 2<\alpha \leq 1,0<\gamma \leq 2 \alpha-1$ and $\gamma<2(1-\alpha)$, then

$$
\delta=\frac{\gamma}{2}<\frac{1}{4}
$$

Proof. Let $\varepsilon_{0}:=\varepsilon /\left\|\frac{d f}{d u}\left(u_{0}\right)\right\|_{L^{\infty}\left(\mathbf{R}^{d}\right)}$. Note that

$$
\left\|u^{h}(0, \cdot)-u_{0}\right\|_{L^{1}\left(\mathbf{R}^{d}\right)} \leq h T V\left(u_{0}\right) .
$$

Combining the basic approximation result (20) with inequalities (21) - (25) and (30) and minimizing over $M$ and $\varepsilon$, one obtains for all $t \in[0, T]$ that

$$
\begin{aligned}
\left\|u^{h}(t, \cdot)-u(t, \cdot)\right\|_{L^{1}\left(\mathbf{R}^{d}\right) \leq} & C^{(1)} h^{(1+\gamma / 2-\alpha) / 2}+C^{(2)} h^{\gamma / 2}+C^{(3)} h^{(1+\gamma / 2) / 2} \\
& +C^{(4)} h^{(1+\gamma-\alpha) / 2}+C^{(5)} h^{1 / 2}+C^{(6)} h,
\end{aligned}
$$

where

$$
\begin{aligned}
& C^{(1)}=C T V\left(u_{0}\right)^{1 / 2}\left\|u_{0}\right\|_{L^{2}\left(\mathbf{R}^{d}\right)}^{1 / 2} T^{1 / 4}\left|\Omega_{h}(T)\right|^{1 / 4}, \\
& C^{(2)}=C\left(\left|\Omega_{h}(T)\right|^{1 / 2}+T V\left(u_{0}\right)^{1 / 2} T^{1 / 2}\right) T^{1 / 2}\left|\Omega_{h}(T)\right|^{1 / 2}, \\
& C^{(3)}=C^{(4)}=C T V\left(u_{0}\right)^{1 / 2} T^{1 / 2}\left|\Omega_{h}(T)\right|^{1 / 2}, \\
& C^{(5)}=C T V\left(u_{0}\right)\left(T^{1 / 2}+\left\|u_{0}\right\|_{L^{\infty}\left(\mathbf{R}^{d}\right)}\left|\Omega_{h}(T)\right|^{1 / 2}\right), \\
& C^{(6)}=C T V\left(u_{0}\right)
\end{aligned}
$$


and the constants $C$ are independent of $h, T$ and $u_{0}$. Here the minimizing $\varepsilon$ is larger than $h$ provided that

$$
h \leq h_{0}:=C\left(T\left|\Omega_{h}(T)\right|\right)^{1 / 3}\left(\frac{\left\|u_{0}\right\|_{L^{2}\left(\mathbf{R}^{d}\right)}}{\left\|u_{0}\right\|_{L^{\infty}\left(\mathbf{R}^{d}\right)}}\right)^{2 / 3} .
$$

The statements of the theorem follow directly from (31).

Finally, we compare conditions (14) and (15). In [8], the author proved convergence under the still weaker assumption that

$$
\lim _{h \rightarrow 0} \frac{h^{2 \alpha}}{\tau}=0
$$

and discussed in detail that this assumption allows irregular families of grids, where cells may become flat with a certain rate as $h \rightarrow 0$. Indeed, this rate is shown to be the optimal rate which assures convergence of general finite volume E-schemes. In the context of the present paper, condition (14) may be guaranteed by the assumptions that there is a constant $C_{4}>0$ such that

$$
\tau \geq \frac{C_{4}}{C_{3}} \rho
$$

and

$$
\frac{h^{2 \alpha}}{\rho} \leq C_{4} h^{\gamma}
$$

For grids $\mathcal{T}_{h}$ consisting of convex polygons, it is shown in [8] that

$$
\frac{\rho_{K}}{2 d} \leq \frac{|K|}{p_{K}} \leq \frac{\rho_{K}}{2} \text { for all } K \in \mathcal{T}_{h} .
$$

Therefore, the CFL-condition (13) is satisfied if

$$
\tau \leq \rho /\left(2 d L_{g}\right),
$$

so condition (33) is compatible with (13). Condition (34) can be rewritten as

$$
\frac{h}{\rho} \leq C_{4} h^{-\beta}, \text { where } \beta:=2 \alpha-1-\gamma .
$$

We call $\beta$ the irregularity coefficient of the family of triangulations $\left(\mathcal{T}_{h}\right)_{h>0}$. If $\beta>0$, then (35) is weaker than (15), and cells may become flat with rate $\beta$ as the grid parameter $h$ tends to zero. Such families of grids are important in applications, in particular when refining the grid in a shock or boundary layer (see for example [1]).

Suppose now that, for one reason or another, we decide to compute on an irregular family of grids with prescribed irregularity coefficient $\beta>0$. It follows from statements (ii) and (iii) of Theorem 3.1 that in order to obtain the convergence rate $\delta$ which is optimal for that given $\beta$, we need to choose

$$
\alpha \geq \frac{\beta+3}{4}
$$

and

$$
\gamma=2 \alpha-1-\beta
$$

From (28), we obtain the convergence rate

$$
\delta=\frac{1-\beta}{4} .
$$


For a regular family of grids, where $\beta=0$, we obtain from statement (i) of Theorem 3.1 that the optimal convergence rate $\delta$ equals $1 / 4$. These relations are illustrated in Figure 1: the parameter regimes identified in statements (i) - (iii) of Theorem 3.1 are labeled by I - III, respectively. In region I, which contains only regular families of grids, we obtain the optimal convergence rate $1 / 4$, and in region II, which admits irregular families of grids, we obtain the rate $(1-\beta) / 4$, which is also optimal. In region III, we obtain convergence, but not with the optimal rate. The lower convergence rate in this region may be understood when comparing the isolines of $\beta$ and $\delta$ in regions II and III.
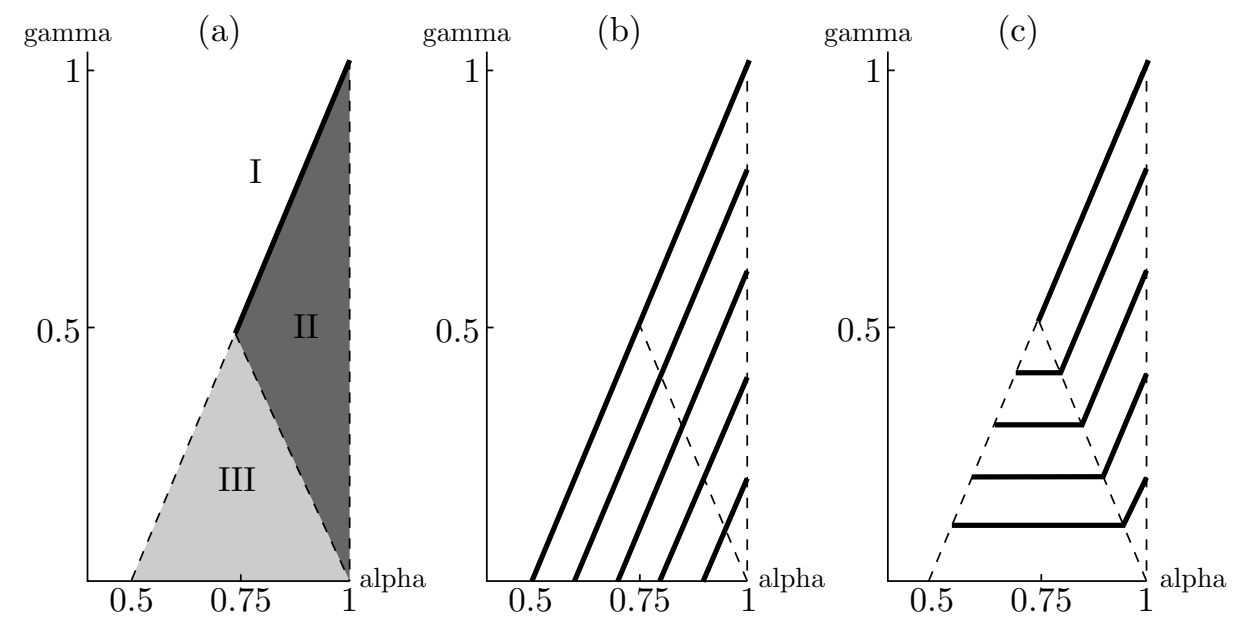

Figure 1. (a) Convergence region for higher-order finite volume schemes in the $\alpha-\gamma$ parameter plane. (b) Irregularity of the grids: isolines of $\beta$. (c) Convergence rates: isolines of $\delta$

The author is grateful to one of the referees for the following remark. In $[4$, Corollary 2.1c], Cockburn and Gremaud prove the estimate

$$
\left\|u_{h}(T)-u(T)\right\|_{L^{1}\left(\mathbf{R}^{d}\right)} \leq\left\|u_{h}(0)-u_{0}\right\|_{L^{1}\left(\mathbf{R}^{d}\right)}+C\left(\frac{\sigma h}{\bar{c}}\right)^{\frac{1}{4}}
$$

for the shock-capturing discontinuous Galerkin method. Here,

$$
\begin{gathered}
\sigma:=\sup _{K} \frac{h_{K}}{\rho_{K}}, \\
\bar{c}:=\inf _{K} \frac{h_{K}}{h} .
\end{gathered}
$$

Translating this to our notation and assuming equality in (35), one obtains

$$
C_{4} h^{-\beta}=\frac{h}{\rho}=\sup _{K} \frac{h}{\rho_{K}} \leq \sup _{K} \frac{h_{K}}{\bar{c} \rho_{K}}=\frac{\sigma}{\bar{c}} .
$$

Therefore, our result (36) implies that the higher-order finite volume methods considered in this paper converge with rate $(\sigma h / \bar{c})^{1 / 4}$ as in [4]. 


\section{ACKNOWLEDGEMENTS}

I would like to thank Professors Bernardo Cockburn and Philippe LeFloch for making preliminary versions of their work available to me before publication. Moreover, I would like to thank Professor Dietmar Kröner and Dr. Mirko Rokyta for the collaboration leading to [6] and for fruitful discussions on the content of this paper.

\section{REFERENCES}

1. R. Beinert, D. Kröner, Finite volume methods with local mesh alignment in 2D, Notes Numer. Fluid Mech. 46 (1994), 38-53.

2. B. Cockburn, F. Coquel, and P. LeFloch, An error estimate for finite volume methods for multidimensional conservation laws, Math. Comp. 63 (1994), 77-103. MR 95d:65078

3. B. Cockburn, F. Coquel, and P. LeFloch, Convergence of the finite volume method for multidimensional conservation laws, SIAM J. Numer. Anal. 32 (1995), 687-705. CMP 95:13

4. B. Cockburn and P. A. Gremaud, Error estimates for finite element methods for scalar conservation laws, To appear in SIAM J. Numer. Anal.

5. F. Coquel and P. LeFloch, Convergence of finite difference schemes for conservation laws in several space dimensions: the corrected antidiffusive flux approach, Math. Comp. 57 (1991), 169-210. MR 91m:65229

6. D. Kröner, S. Noelle, and M. Rokyta, Convergence of higher-order upwind finite volume schemes on unstructured grids for scalar conservation laws in several space dimensions, $\mathrm{Nu}-$ mer. Math. 71 (1995), 527-560. CMP 96:02

7. N. Kuznetsov, Accuracy of some approximate methods for computing the weak solutions of a first-order quasi-linear equation, USSR Comp. Math. and Math. Phys. 16 (1976), 105-119.

8. S. Noelle, Convergence of higher-order finite volume schemes on irregular grids, Adv. Comp. Math. 3 (1995), 197-218. MR 95:10

9. S. Osher, Riemann solvers, the entropy condition and difference approximations, SIAM J. Numer. Anal. 21 (1984), 217-235. MR 86d:65119

10. J. Vila, Convergence and error estimates in finite volume schemes for general multidimensional scalar conservation laws: I. Explicit monotone schemes, Math. Modelling Numer. Analysis 28 (1994), 267-295. CMP 94:12

Institute of Applied Mathematics, Wegeler Str. 10, 53115 Bonn, Germany

E-mail address: noelle@iam.uni-bonn.de 